\title{
Reflets
}

Revue d'intervention sociale et communautaire

\section{La femme, sa sexualité et son pouvoir sexuel Un programme d'appropriation de sa sexualité}

\section{Ina Motoi}

Volume 19, numéro 1, printemps 2013

URI : https://id.erudit.org/iderudit/1018048ar

DOI : https://doi.org/10.7202/1018048ar

Aller au sommaire du numéro

Éditeur(s)

Reflets, Revue d'intervention sociale et communautaire

ISSN

1203-4576 (imprimé)

1712-8498 (numérique)

Découvrir la revue

Citer cet article

Motoi, I. (2013). La femme, sa sexualité et son pouvoir sexuel : un programme d'appropriation de sa sexualité. Reflets, 19(1), 238-242.

https://doi.org/10.7202/1018048ar d'utilisation que vous pouvez consulter en ligne.

https://apropos.erudit.org/fr/usagers/politique-dutilisation/ 


\title{
La femme, sa sexualité et son pouvoir sexuel
}

\section{Un programme d'appropriation de sa sexualité}

\author{
Ina Motoi \\ Professeure en travail social, Université du Québec en \\ Abitibi-Témiscamingue
}

«Il n’y a pas plus efficace qu'une femme qui est écœurée... » ${ }^{1}$

La femme, sa sexualité et son pouvoir sexuel - Programme d'appropriation de sa sexualité (Motoi et Dufour, 2011) est le fruit de la mise en commun de plusieurs expériences d'intervention et de deux recherches narratives, la première amorcée par Dufour avec le Projet intervention prostitution Québec (PIPQ) (voir Dufour, 2004) et poursuivie à la Maison de Marthe à Québec, « un lieu d'accueil et un mode d'intervention pour les femmes qui désirent quitter la prostitution ", et la seconde entreprise par Motoi en 2004 en Abitibi ${ }^{2}$. Les femmes rencontrées dans ces différents contextes ont accepté de partager leur méthode de connaissance de leur vécu sexuel afin d'aider d'autres femmes qui se retrouvaient seules, tout comme elles, sur le même chemin de l'objectification sexuelle. Elles nous ont appris comment elles construisaient leur connaissance de leur vécu sexuel et comment cette démarche, construite dans et par la sexualité, les a fait cheminer vers l'appropriation de cette dernière et de leur pouvoir sexuel.

Afin de transmettre cette méthode de connaissance développée par les femmes participantes, Dufour et moi-même avons bâti ce programme visant à faciliter une réflexion, entre femmes, sur l'appropriation de la sexualité. Il s'agit d'une méthode dialogique 
"...la recherche de pouvoir sexuel des participantes catalyse leur réflexion personnelle et leur réflexion entre femmes et facilite leurs démarches d'appropriation de leur sexualité. » d'intervention de groupe dont l'objectif est de donner une voix aux multiples petits récits des femmes plutôt qu'aux grands récits médiatisés sur la prostitution et la sexualité.

Les femmes dialoguant en groupe font habituellement le lien entre sexualité et prostitution, cette double problématique se retrouvant au cœur de leur vécu sexuel. Par conséquent, la sexualité devient le point de référence pour parler de violence sexuelle (inceste, agression sexuelle, prostitution, etc.). De plus, se sentir utilisée comme une prostituée, ce rapport individuel au contexte de prostitution et au prostitueur, dans l'espace public ou dans un couple, est mis de l'avant comme rapport prostitutionnel. C'est pourquoi, pour se sortir de ces situations d'impuissance sexuelle, la recherche de pouvoir sexuel des participantes catalyse leur réflexion personnelle et leur réflexion entre femmes et facilite leurs démarches d'appropriation de leur sexualité.

La connaissance construite par chaque femme rencontrée pour elle-même, afin d'alléger sa propre souffrance en dégageant de la cohérence à partir de son vécu sexuel, s'est développée à partir de distinctions fondamentales qu'elle a du faire entre sexualité et rapports prostitutionnels et entre sexualité et prostitution. Dans le groupe de femmes, cette connaissance se trouve prise en considération, ainsi que se trouve reconnue leur liberté de définir elles-mêmes leur propre vécu. Chaque femme est maîtresse de sa propre démarche pour devenir sujet de sa vie sexuelle et de son existence. Pour le demeurer, elle choisit de ne pas oublier ce que c'est d'être objet sexuel. C'est pourquoi la plupart des participantes identifient au centre de leur réflexion et de leur transformation une tension principale entre être sujet de sa sexualité et être objet sexuel.

La femme, sa sexualité et son pouvoir sexuel - Programme d'appropriation de sa sexualité permet de faire reconnaître à l'aide $\mathrm{du}$ dialogue le pouvoir de chaque participante à construire du sens dans sa vie sexuelle. Les femmes dépassent ainsi leur propre doute par rapport à leur capacité de donner et de construire du sens, en toute subjectivité, à ce qui se rapporte à leur vécu sexuel. Les participantes détiennent et tissent leurs propres réponses et leurs propres solutions, qu'elles aient vécu de la violence sexuelle 
ou qu'elles souhaitent seulement réfléchir sur leur sexualité et partager leur réflexion avec d'autres femmes.

Le programme est construit sous la forme de quatre rencontres de trois heures chacune. Les objectifs ${ }^{6}$ du programme sont :

1. S'orienter dans et par rapport à son tvécu sexuel;

2. Réaffirmer ensemble comme femmes la frontière entre sexualité et rapport prostitutionnel, frontière que chacune doit tracer en soi pour s'approprier sa sexualité;

3. Reconnaitre sa propre méthode de connaissance pour retrouver son pouvoir sexuel;

4. Transformer sa résistance en pouvoir sexuel. (Motoi et Dufour, 2011, p. 5)

\section{Une démarche en quatre étapes}

La démarche proposée dans ce programme met de l'avant quatre étapes. Lors de la première, la femme ouvre ou consolide un espace dialogique en soi, où elle se parle à elle-même, sans que personne d'autre ne puisse lui dire ce qu'elle vit. "Le dialogue intérieur, souverain en soi, est souvent leur seul espace intime dans une vie sexuelle occupée par d'autres » (Motoi et Dufour, 2011,

«La participante

y exprime souvent sa résistance à son objectification sexuelle..."

\section{"Elle s'approprie cette perspective et se positionne de façon conflictuelle par rapport à son statut de femme- objet...»} p.17). Elle peut ainsi réfléchir, reconnaitre, nommer et (re)définir ce qu'elle vit et ce qu'elle ne veut plus vivre. La participante y exprime souvent sa résistance à son objectification sexuelle, si c'est le cas, et reconnaît ses propres valeurs et ses propres limites.

À la deuxième étape, la femme identifie les tensions et les contradictions qu'elle vit ou qu'elle ne veut pas vivre en tant que femme-objet. Elle veut comprendre, sans accepter, le vécu de femme-objet. Elle saisit ainsi sa propre perspective de femme-sujet de sa sexualité sur son vécu de femme-objet. Elle s'approprie cette perspective et se positionne de façon conflictuelle par rapport à son statut de femme-objet, en commençant, par essais et par erreur, à transformer sa résistance en pouvoir sexuel. Au cœur de ce cheminement, la femme s'impose la notion de limite, celle qu'elle décide de poser, comme frontière entre ce qu'elle aime et ce qu'elle n'aime pas. Alors, une tension principale apparait entre deux vécus sexuels qui peuvent aussi se superposer (Motoi et Dufour, 2011, p. 81-84) : 
1. je n'aime pas être un objet sexuel (je n'aime pas faire plaisir à l'Autre ou dépasser mes limites, ce n'est pas valorisant pour moi et je vis mon impuissance);

2. mais, je me pose comme objet sexuel (je ne me soucie pas de moi, je me marchande, cela me donne le pouvoir de l'objet sexuel).

La troisième étape consiste pour la femme à reconnaitre son pouvoir sexuel, cette puissance en devenir, pour bâtir ou consolider son savoir de femme-sujet.

"Quand tu vas venir à voir arriver cette situation-là, tu vas savoir que si tu t'affirmes pas tout de suite, là, tu vas tomber dans l'ancien pattern, puis..." (Une participante partageant son savoir, dans Motoi et Dufour, 2011, p.103)

La femme comme sujet de sa propre sexualité identifie sa méthode distinctive de connaissance de son vécu sexuel, les stratégies qu'elle utilise et les objectifs qu'elle se donne. Pour cela, chacune a posé en elle le pouvoir de dialoguer avec soi-même comme un pouvoir de construire sa théorie personnelle de ce qu'elle vit sexuellement, qui s'autovérifie et s'érige comme un savoir dont elle évalue assidument la cohérence et la pertinence.

La quatrième étape du programme est plus pédagogique. À partir des données de recherche de Dufour (2004) et d'autres de ses données inédites, on présente comment on en vient à se prostituer, et cela, à partir de quels systèmes producteurs de prostitution. Cela permet de comprendre la réalité de la prostitution et ses effets sur son vécu sexuel et de se situer par rapport à eux. Les participantes réfléchissent sur la sexualisation précoce de la jeune

"Elles sont dans leurs propres vies en mesure de s'approprier leur sexualité et de faire ainsi une différence significative comme femmes-sujets. » fille qui en vient à se prostituer. Elles essaient de saisir les choix que les femmes de leur lignée qui les ont précédées ont du faire en ce qui concernait leur propre vécu sexuel. Elles sont dans leurs propres vies en mesure de s'approprier leur sexualité et de faire ainsi une différence significative comme femmes-sujets.

Depuis que La femme, sa sexualité et son pouvoir sexuel - Programme d'appropriation de sa sexualité a été publié en septembre 2011, 
"Les participantes à nos recherches proposent à travers ce programme un cadre de réflexion qui peut renouveler les pratiques actuelles d'intervention..." cinquante-sept intervenantes venant de différents organismes de femmes ont déjà été formées au Québec. Elles ont appris à adapter au vécu et à la perspective de chaque femme participant aux quatre séances les points de repère identifiés par celles qui ont collaboré aux recherches initiales. Le programme s'offre comme un cadre de réflexion qui respecte le rythme, la dynamique et les enjeux de chaque groupe de femmes. On ne cherche pas le consensus à tout prix. Les participantes ont fréquemment des perspectives différentes sur un même sujet ou sur un vécu semblable, tout en essayant de se comprendre mutuellement. Le rôle de l'animatrice est de faciliter cette compréhension mutuelle qui prend lieu dans le dialogue entre femmes. Leurs différences participent à leur apprentissage à articuler davantage leur propre théorie de leur vécu sexuel. Dans ces rencontres la communication est essentielle, autant comme dialogue avec soi que comme dialogue, en toute égalité épistémologique, avec les autres femmes, participantes ou animatrices.

Les participantes à nos recherches proposent à travers ce programme un cadre de réflexion qui peut renouveler les pratiques actuelles d'intervention dans le domaine de la violence sexuelle.

\section{Notes}

Participante à la recherche de Motoi, cité dans Motoi et Dufour, 2011, p. 51.

2 Neuf femmes âgées de 20 à 52 ans ont participé à ce groupe et se présentent ainsi lors de l'entrevue de présélection : 5 comme hétérosexuelles, 2 comme lesbiennes, 2 comme bisexuelles, 5 comme survivantes d'agression sexuelle, 2 se retrouvant dans une situation limite à la prostitution, 3 ayant fait de la prostitution.

\section{Bibliographie}

DUFOUR, Rose (2004). Je vous salue... Marion, Carmen, Eddy, Jo-Annie, Nancy, Jade, Lili, Virginie, Marie-Pierre - Le point zéro de la prostitution, Québec, MultiMondes, $646 \mathrm{p}$.

MOTOI, Ina, et Rose DUFOUR (2011). La femme, sa sexualité et son pouvoir sexuel. Programme d'appropriation de sa sexualité, Québec, PUQ, 164 p. 